\author{
ANNALES \\ POLONICI MATHEMATICI \\ XXXIV (1977)
}

\title{
Generic properties of linear control systems depending on a parameter
}

\author{
by M. Medvé (Bratislava, Czechoslovakia)
}

\begin{abstract}
In this paper generic or typical properties of linear control systems depending on a parameter are investigated. We consider a set of parametrized control systems $(\mu) \dot{x}=A(\mu) x+B(\mu) u$, where $A, B$ are $C^{r}$-matrices depending on a parameter $\mu, A$ is $n \times n, B$ is $n \times m$ and this set is endowed with a topology. A property of a system is generic iff "almost all" systems have this property: In this paper the following is proved: If $\operatorname{dim} \mu=1, m=1$, then the set of points $\mu_{0}$ such that the system $\left(\mu_{0}\right)$ is not completely controllable consists generically of isolated points. This set is generically empty for $m>1$. The case $\operatorname{dim} \mu=2$ is also investigated.
\end{abstract}

One of the main themes of the recent work in the qualitative theory is the search for generic, or typical properties of dynamical systems. It is not our aim to describe these properties. These problems are described e.g. in [1]. But such model will serve us as a model for study of typical properties of control systems. This paper is concerned with linear control systems depending on a parameter from the point of view of their generic properties.

Let $M(i, j)$ be the set of all $i \times j$ matrices. Denote by $F^{\prime}=F^{\prime}(n, m)$ the set of all linear control systems $\dot{x}=A x+B u$, where $A \in M(n, n)$. $B \in M(n, m)$. Since this system is uniquely determined by the pair of matrices $A, B$, we shall call it $(A, B)$. The space of such systems, endowed with the topology induced by the metric $d\left[(A, B),\left(A^{\prime}, B^{\prime}\right)\right]=\left|A-A^{\prime}\right|_{1}+$ $+\left|B-B^{\prime}\right|_{2}$, where $|\cdot|_{1}$ is a norm in $M(n, n),|\cdot|_{2}$ is a norm in $M(n, m)$, will also be denoted by $F^{\prime}$ or $F^{\prime}(n, m)$.

Definition 1. A property $G(A, B)$ of a system $(A, B) \in F^{\prime}(n, m)$ is generic in $F^{\prime}(n, m)$ iff the set $\left\{(A, B) \in F^{\prime}(n, m) \mid G(A, B)\right\}$ contains a residual set, i.e., a set which is a countable intersection of open dense subsets of $F^{\prime}(n, m)$.

Remark. The space $F^{\prime}(n, m)$ is complete and therefore by the Baire category theorem every residual subset of $F^{\prime}(n, m)$ is dense in $F^{\prime}(n, m)$.

Definition 2. A system $(A, B) \in F^{\prime}(n, m)$ is called completely controllable iff for every $x_{0}, x_{1} \in R^{n}$ there is a bounded, measurable control 
function $u(t)$ on some finite interval $\left[0, t_{1}\right]$ such that the solution $x(t)$ of $(A, B), \quad x(0)=x_{0}$ under $u=u(t)$ satisfies $x\left(t_{1}\right)=x_{1}$.

Denote by $F_{0}^{\prime}=F_{0}^{\prime}(n, m) \subset F^{\prime}(n, m)$ the set of all completely controllable systems.

Proposition ([6], Theorem 11). The set $F_{0}^{\prime}(n, m)$ is open and dense in $F^{\prime}(n, m)$.

This proposition states that complete controllability is a generic property in $F^{\prime}(n, m)$. We shall show that for control systems depending on a parameter it is not possible to avoid values of parameter in which the system is not completely controllable (not even generically). In the case of 1-dimensional parameter it is not possible if the matrix $B(\mu)$ is $n \times 1$, but it is possible if $B(\mu)$ is $n \times m$, where $m>1$.

Definition 3. Let $A \in C^{r}(P, M(n, n)), B \in C^{r}(P, M(n, m))$, where $P$ is a compact $C^{r}$-manifold. Then the system $\dot{x}=A(\mu) x+B(\mu) u$ is called a linear $C^{r}$-parametrized control system. Since this system is uniquely determined by the pair of mappings $A, B$, we shall call it $(A, B)$. We denote by $F_{P}^{r}(n, m)$ the set of all such systems with the $C^{r}$-topology.

Definition 3. A property $G(A, B)$ of a system $(A, B) \in F_{P}^{r}(n, m)$ is generic in $F_{P}^{r}(n, m)$ iff the set $\left\{(A, B) \in F_{P}^{r}(n, m) \mid G(A, B)\right\}$ contains a residual set.

We can identify $M(n, n)$ with $R^{n^{2}}$. The group $\mathrm{GL}\left(R^{n}\right)$ has a natural action on $R^{n^{2}}$ defined by $(g, A) \rightarrow g A g^{-1}, A \in R^{n^{2}}, g \in \mathrm{GL}\left(R^{n}\right)$. This group decomposes the space $R^{n^{2}}$ into orbits. $E, F \in R^{n^{2}}$ belongs to the same orbit iff $F=h E h^{-1}$ for some $h \in \mathbf{G L}\left(R^{n}\right)$.

A decomposition of the space $R^{n^{2}}$ into a finite number of smooth manifolds is called a stratification of $R^{n^{2}}$.

Let us decompose the space $M(n, n)$ into clases of matrices with Jordan's blocks of the same dimension, which differ in their eigenvalues only. This decomposition is a stratification of $M(n, n)$, i.e., $M(n, n)=\bigcup_{i=0}^{q} N_{i}$, where $N_{i}, i=0,1, \ldots, q$ are disjoint submanifolds of $M(n, n)$ (strata). By [2] the codimension of such a stratum is

$$
c=d-v=2 \sum_{i=1}^{j}\left(n_{2}(i)+2 n_{\mathrm{s}}(i)+\ldots\right)+\sum_{i=1}^{\nu}\left(n_{1}(i)+n_{2}(i)+\ldots\right)-v,
$$

where $d$ is the dimension of the orbit corresponding to this stratum and $n_{1}(i) \geqslant n_{2}(i) \geqslant \ldots$ are the dimensions of Jordan's blocks corresponding to different eigenvalues $\lambda(i)(i=1,2, \ldots, v)$.

Remark. If all eigenvalues are simple, then $v=n, n_{1}(i)=1$ for $i=1,2, \ldots, n, n_{k}(i)=0$ for $k>1$ and $c=0$. Therefore the stratum corresponding to a matrix with simple eigenvalues has codimension 0 . 
Let $N_{0}$ denote this stratum. A matrix belonging to a stratum of codimension $c=1$ has only one eigenvalue of multiplicity 2 with corresponding Jordan's block of the type

$$
a^{2}:\left(\begin{array}{ll}
a & 1 \\
0 & \alpha
\end{array}\right) .
$$

Call this stratum $N_{1}$. There are only two strata of codimension 2:

1. The stratum corresponding to matrices with one Jordan's block of the type

$$
a^{3}:\left(\begin{array}{lll}
a & 1 & 0 \\
0 & a & 1 \\
0 & 0 & a
\end{array}\right)
$$

and with all other Jordan's blocks corresponding to simple eigenvalues. Call this stratum $N_{2}$.

2. The stratum corresponding to matrices with Jordan's block of the type

$$
\alpha^{2} \beta^{2}:\left(\begin{array}{cccc}
\alpha & 1 & 0 & 0 \\
0 & \alpha & 0 & 0 \\
0 & 0 & \beta & 1 \\
0 & 0 & 0 & \beta
\end{array}\right), \quad \alpha \neq \beta
$$

and with all other Jordan's blocks corresponding to simple eigenvalues. Call this stratum $N_{3}$.

1. One-parameter systems. Let $M(n, n)=\bigcup_{i=0}^{q} N_{i}$ be the stratification of $M(n, n)$. Denote by $F_{0}^{r}(n, m)$ the set of $(A, B) \in F_{P}^{r}(n, m)$ for which $A \bar{\cap} N_{i}$ for $i=1,2, \ldots, q$. ( $A \bar{\cap} N_{i}$ means that the mapping $A$ transversally intersects the manifold $N_{i}$; see [1] or [6].)

From [5], [6], [2] we obtain the following lemma.

LeMma 1. If $\operatorname{dim} P=p, r>\max (0, p-c)$, where $c=\operatorname{codim} N_{i}$, then the set $\left\{A \in C^{r}(P, M(n, n)) \mid A \bar{\cap} N_{i}\right\}$ is open and dense in $C^{r}(P, M(n, n))$.

LEMMA 2. If $r \geqslant p$, then the set $F_{0}^{r}(n, m)$ is open and dense in $F_{P}^{r}(n, m)$.

This is an immediate consequence of Lemma 1 .

Remark. If $A \bar{\cap} N_{i}$, then by [1], Corollary 17.2, the set $K_{i}(A)$ $=A^{-1}\left(N_{i}\right)$ is a submanifold of $P$ and $\operatorname{codim} K_{i}(A)=\operatorname{codim} N_{i}$.

If $\operatorname{dim} P=1$ and $(A, B) \in F_{0}^{r}(n, m)$, then by [1], Corollary 17.2, 
$A(P) \cap\left(\bigcup_{i=2}^{q} N_{i}\right)=\emptyset$ and $\operatorname{codim} K_{i}(A)=i$ for $i=0,1$, i.e., $\operatorname{dim} K_{0}(A)=1$, $\operatorname{dim} K_{1}(A)=0$.

Lemma 3 ([4], Lemma 6). Let $F \in C^{r}(P, M(n, n)), \mu_{0} \in P$ and suppose that $F\left(\mu_{0}\right)$ has simple eigenvalues $\lambda_{j}^{0}=\lambda_{1 j}^{0}+i \lambda_{2 j}^{0}, j=1,2, \ldots, k, \lambda_{k+1}^{0}, \ldots, \lambda_{n}^{0}$. Then there are: a chart $(U, \alpha)$ on $P$ at $\mu_{0}$, a neighbourhood $N$ of $v_{0}=\alpha^{-1}\left(\mu_{0}\right)$ in $V=a^{-1}(U)$, and for every $j=1,2, \ldots, n$ a unique $C^{r}$-function $\lambda_{j}$ : $N \rightarrow C$ such that $\lambda_{j}\left(\nu_{0}\right)=\lambda_{j}^{0}$ and $\lambda_{j}(v)$ is an eigenvalue of the matrix $\tilde{F}(v)$ $=F \cdot \alpha^{-1}(v)$ for every $v \in V$. Further, there is a $C^{r}$-matrix $G$ on $V$ such that the matrix $D(v)=G^{-1}(v) \tilde{F}(v) G(v)$ has the form

$$
D=\operatorname{diag}\left\{\left(\begin{array}{ll}
\lambda_{11} & \lambda_{21} \\
-\lambda_{21} & \lambda_{11}
\end{array}\right), \ldots,\left(\begin{array}{cc}
\lambda_{1 k} & \lambda_{2 k} \\
-\lambda_{2 k} & \lambda_{1 k}
\end{array}\right), \lambda_{k+1}, \ldots, \lambda_{n}\right\},
$$

where $\lambda_{j}=\lambda_{1 j}+i \lambda_{2 j}, j=1,2, \ldots, k$.

Define the mapping $H_{B}^{i}: V \rightarrow R^{n}, H_{B}^{i}(v)=G^{-1}(\nu) b_{i}(v)$, where $b_{i}(\nu)$ is the $i$-th column of the matrix $B \cdot \alpha^{-1}(\nu)$. Let

$$
W_{i_{1}, \ldots, i_{k}}=\left\{\left(y_{1}, \ldots, y_{i_{1}}, \ldots, y_{i_{k}}, \ldots, y_{n}\right) \in R^{n} \mid y_{i_{1}}=0, \ldots, y_{i_{k}}=0\right\} \text {. }
$$

Let $W=\bigcup_{I} W_{i_{1}, \ldots, i_{k}}, \quad$ where $\quad I=\left\{\left(i_{1}, \ldots, i_{k}\right) \mid 1 \leqslant k \leqslant n, \quad i_{1}, \ldots, i_{k} \in\right.$ $\in\{1,2, \ldots, n\}\}$. The sets $W_{i_{1}, \ldots, i_{k}}$ are smooth submanifolds of $R^{n}$ and $\operatorname{codim} W_{i_{1}, \ldots, i_{k}} \geqslant 1$ for all $\left(i_{1}, \ldots, i_{k}\right) \in I$.

DEFINITION 4. $F_{1}^{r}(n, m)$ is the set of $(A, B) \in F_{0}^{r}(n, m)$ with the following property: If $\mu_{0} \in P-K_{1}(A)$ and $(U, \alpha)$ is a chart on $P$ at $\mu_{0}$ as in Lemma 3 , then $H_{B}^{i} \bar{\cap} W$ for all $i=1,2, \ldots, m$.

LEMMA 4. If $\operatorname{dim} P=1$ and $r \geqslant 1$, then the set $F_{1}^{r}(n, m)$ is residual in $F_{P}^{r}(n, m)$.

For the proof of this lemma we need two further lemmas.

LEMMA 5. Let $r \geqslant 1$ and let $V_{1} \subset V$ be a closed subset of $V$. Then the set

$$
H_{i_{1}, \ldots, i_{k}}\left(V_{1}\right)=\left\{B \in C^{r}(P, M(n, m)) \mid \tilde{H}_{B}^{i} \bar{\cap} W_{i_{1}, \ldots, i_{k}}, i=1,2, \ldots, m\right\}
$$

is open and dense in $C^{r}(P, M(n, m))$, where $\tilde{H}_{B}^{i}$ is the restriction of $B_{B}^{i}$ to $V_{1}$.

Proof. Openness follows from [1], Theorem 18.2. We shall prove density. Define the mapping $\varrho_{i}: C^{r}(P, M(n, m)) \rightarrow C^{r}(V, M(n, 1))$, $\varrho_{i}(B)=H_{B}^{i}$ for $B \in C^{r}(P, M(n, m)) \cdot e v_{Q_{i}}: C^{r}(P, M(n, m)) \times V \rightarrow M(n, 1)$, $e v_{Q_{i}}(B, v)=H_{B}^{i}(v)=G^{-1}(v) b_{i}(v)$. Since the matrix $G^{-1}(v)$ is regular, then $e v_{Q_{i}} \vec{\cap} Q$ for every submanifold $Q$ of $M(n, 1)$. The assumptions of [1], Theorem 19.1, are fulfilled and so the set

$$
\tilde{H}_{i_{1}, \ldots, i_{k}}(V)=\bigcap_{i=1}^{m}\left\{B \in C^{r}(P, M(n, 1)) \mid H_{B}^{i} \bar{\cap} W_{i_{1}, \ldots, i_{k}}\right\}
$$

is dense in $C^{r}(P, M(n, 1))$; therefore the set $H_{i_{1}, \ldots, i_{k}}\left(V_{1}\right)$ is dense, too. 
Definimion 5. $F_{1 l}^{r}(n, m)$ ( $l$ is a natural number) is the set of $(A, B) \in$ $\in F_{0}^{r r}(n, m)$ with the following property: If $\mu_{0} \in P-D_{l}$, where $D_{l}=\{\mu \in P \mid$ $\left.d\left(\mu, K_{1}(A)\right)<1 / l\right\}, d$ is a metric on $P$ and $(U, a)$ is a chart on $P$ at $\mu_{0}$ as in Lemma 3 , then $H_{B}^{i} \bar{\cap} W$ for $i=1,2, \ldots, m$.

It is obvious that this definition is independent on the chart $(U, a)$ and $F_{1}^{r}(n, m)=\bigcap_{l=1}^{\infty} F_{1 l}(n, m)$.

LEMMA 6. If $\operatorname{dim} P=1$ and $r \geqslant 1$, then the set $F_{1 l}^{r}(n, m)$ is open and dense in $F_{0}^{r}(n, m)$.

Proof. Openness follows from [1], Theorem 18.2. We shall prove density. Let $(A, B) \in F_{0}^{r}(n, m)$. The set $P-D_{l}$ is compact and therefore we can cover it by a finite number of charts $\left\{\left(U_{\sigma} ; a_{\sigma}\right)\right\}_{\sigma=1}^{s}$, which are such that $a_{\sigma}\left(U_{\sigma}\right) \cap a_{\sigma+1}\left(U_{\sigma+1}\right) \neq \emptyset$ for $\sigma=1,2, \ldots, s-1$ and $a_{\sigma}\left(U_{\sigma}\right) \cap \alpha_{\sigma+j}\left(U_{\sigma}\right)$ $=\varnothing$ for $j>1$. Now we proceed by induction with respect to $\sigma$. If $\sigma=1$, then by Lemma 5 there is a system $(A, \tilde{B}) \in F_{P}^{r}(n, m)$ sufficiently close to the system $(A, B)$ such that if $\mu_{0} \in\left(P-D_{l}\right) \cap U_{l}$, then $H_{\vec{B}}^{i} \bar{\cap} W$ for $i=1,2, \ldots, m$. Suppose $H_{B}^{i} \bar{\cap} W$ for $i=1,2, \ldots, m$ on $\bigcup_{k=1}^{\sigma} \alpha_{k}^{-1}\left(U_{k}\right)$, $\sigma>1$. We shall prove that there is a system $(A, \hat{B}) \in \underset{\sigma+1}{F_{P}^{r}(n, m) \text { sufficiently }}$ close to the system $(A, B)$ such that if $\mu_{0} \in\left(P-D_{l}\right) \cap\left(\bigcup_{k=1}^{\sigma+1} U_{k}\right)$, then $H_{\hat{B}}^{i} \bar{\cap} W$ on $\bigcup_{k=1}^{\sigma+1} \alpha_{k}^{-1}\left(U_{k}\right)$. By Lemma 5 there is a $\operatorname{system}(A, \hat{B}) \in F_{P}^{r}(n, m)$ sufficiently close to the system $(A, \tilde{B})$ such that if $\mu_{0} \in\left(P-D_{l}\right) \cap U_{\sigma+1}$, then $H_{\hat{B}}^{i} \bar{\cap} W$ for $i=1,2, \ldots, m$ on $a^{-1}\left(U_{\sigma+1}\right)$. Then by the induction assumption and by the openness of the transversality condition we also have $H_{\hat{B}}^{i} \bar{\cap} W$ for $i=1,2, \ldots, m$ on $\bigcup_{k=1}^{\sigma+1} \alpha^{-1}\left(U_{k}\right)$, and this completes the proof.

Since $F_{1}^{r}(n, m)=\bigcap_{i=1}^{\infty} F_{1 l}(n, m)$, the proof of Lemma 4 follows from Lemma 2 and Lemma 6.

Corollary to Lemma 4. Let $(A, B) \in F_{1}^{r}(n, m), \mu_{0} \in P-K_{1}(A)$ and let $(U, \alpha)$ be a chart on $P$ at $\mu_{0}$ as in Lemma 3. Then by [1], Corollary 17.2, the set $\left\{\mu \in \alpha^{-1}(U) \mid H_{B}^{i}\left(\alpha^{-1}(U)\right) \cap W \neq \varnothing\right.$ for $\left.i=1,2, \ldots, m\right\}$ consists of isolated points.

Proof. codim $W_{i_{1}, \ldots, i_{k}} \geqslant 1, H_{B}^{i} \bar{\cap} W_{i_{1}, \ldots, i_{k}}$ and therefore by [1], Corollary 17.2, codim $\left[\boldsymbol{H}_{B}^{i}\right]^{-1}\left(W_{i_{1}, \ldots, i_{k}}\right)=\operatorname{codim} W_{i_{1}, \ldots, i_{k}} \geqslant 1$, i.e., $\operatorname{dim}\left[H_{B}^{i}\right]^{-1}\left(W_{i_{1}, \ldots, i_{k}}\right)<1$. Since at least one of the manifolds $W_{i_{1}, \ldots, i_{k}}$ has codimension 1 , the proof is complete.

Lemma 7. Let $(A, B) \in F^{\prime}(n, 1)$, where $A=\operatorname{diag}\left\{A_{1}, A_{2}, \ldots, A_{n}\right\}$ is the Jordan form of the matrix $A$ and let $B=\operatorname{transp}\left(b_{1}, b_{2}, \ldots, b_{n}\right)$, where $b_{i} \neq 0$ for $i=1,2, \ldots, n$. Suppose that every two blocks of the matrix $A$ 
have no common eigenvalues. Then the system $(A, B)$ is completely controllable.

THEOREM 1. Let $\operatorname{dim} P=1$ and $r \geqslant 1$. Then there is a subset $F_{2}^{r}(n, m)$ of $F_{P}^{r}(n, m)$ residual in $F_{P}^{r}(n, m)$ such that the following is true: If $(A, B) \in$ $\in F_{2}^{r}(n, m)$, then for $m=1$ the set $R(A, B)$ consists of isolated points only and if $m>1$, then $R(A, B)=\emptyset$.

Remark. By [5], Theorem 5, II. $\S 2,(A(\mu), B(\mu)) \in F^{\prime}(n, m)$ is completely controllable if and only if rank $\left(B(\mu), A(\mu) B(\mu), \ldots, A^{n-1}(\mu) B(\mu)\right)$ $=n$. Therefore if $(A, B) \in F_{2}^{r}(n, m)$, then the set $R(A, B)$ consists of points $\mu_{0}$ for which the following is true:

If $G^{-1}\left(\mu_{0}\right) A\left(\mu_{0}\right) G\left(\mu_{0}\right)=A\left(\mu_{0}\right)=\operatorname{diag}\left(A_{1}, A_{2}, \ldots, A_{k}\right)$ is the Jordan form of the matrix $A\left(\mu_{0}\right)$, then the matrix $B\left(\mu_{0}\right)$ has no columns $b_{i}=$ $\operatorname{transp}\left(b_{i 1}, b_{i 2}, \ldots, b_{i n}\right)$ such that $b_{i, e_{1}} \neq 0, b_{i, e_{1}+e_{2}} \neq 0, \ldots, b \underset{i, \sum_{j=1}^{k} e_{j}}{k} \neq 0$, whero $e_{j}(j=1,2, \ldots, k)$ is the dimension of the block $A_{j}$. If the matrix $B(\mu)$ has one column only, then we can not avoid such value $\mu_{0}$ of the parameter $\mu$. If the matrix $B(\mu)$ has at least two columns, this is possible.

Proof of Theorem 1. By Lemma 6 the set $F_{1 l}^{r}(n, m)$ is open and dense in $F_{P}^{r}(n, m)$. If $(A, B) \in F_{1 l}^{r}(n, m)$, then by Corollary to Lemma 2 the matrix $A(\mu)$ has all eigenvalues of multiplicity $\leqslant 2$ for all $\mu \in P$ and the set $K_{1}(A)$ of such $\mu \in P$ for which the matrix $A(\mu)$ has an eigenvalue of multiplicity 2 consists of isolated points. Therefore, by Lemma 7, if $m=1$, it suffices to put $F_{2}^{r}(n, m)=F_{1}^{r}(n, m)=\bigcap_{l=1}^{\infty} F_{1 l}(n, m)$. Suppose $m>1$. Denote by $F_{21}^{r}(n, m)$ the set of all $(A, B) \in F_{1 l}^{r}(n, m)$ such that if $b_{1}(\mu), b_{2}(\mu), \ldots, b_{m}(\mu)$ are columns of $B(\mu)$, then $\left(A, b_{1}\right) \in F_{1 l}^{r}(n, 1)$, $\left(A, b_{2}\right) \in F_{1 l}^{r}(n, 1)$ and $R\left(A, b_{1}\right) \cap R\left(A, b_{2}\right)=\emptyset$. Since in the case $m=1$ Theorem 1 has been proved, the sets $R\left(A, b_{1}\right), R\left(A, b_{2}\right)$ consist of isolated points. Therefore we can achieve by arbitrarily small perturbation $\tilde{b}_{i}$, $i=1,2$, of $b_{i}, i=1,2$, that $R\left(A, \tilde{b}_{1}\right) \cap R\left(A, \tilde{b}_{2}\right)=\emptyset$. Since the set $F_{l l}^{r}(n, 1)$ is open and dense in $F_{p}^{r}(n, 1)$, the set $F_{2 l}^{r}(n, m)$ is open and dense in $F_{P}^{r}(n, m)$ and it suffices to put $F_{2}^{r}(n, m)=\bigcap_{l=1}^{\infty} F_{2 l}^{r}(n, m)$. It is clear that if $(A, B) \in F_{2}^{r}(n, m)$, then $R(A, B)=\emptyset$.

2. Two-parametric systems. Let $\operatorname{dim} P=2$ and $(A, B) \in F_{0}^{r}(n, m)$. Then $A^{-1}\left(N_{1}\right)$ is a 1-dimensional submanifold of $P$ and so blocks of the form $a^{2}$ occur generically on a 1-dimensional set of parameters. Since $\operatorname{dim} A^{-1}\left(N_{2}\right)=0$, blocks of the form $a^{3}$ or blocks $a^{2} \beta^{2}$ occur generically at isolated points of $P$. Therefore, analogously to the case $\operatorname{dim} P=1$, we can achieve complete controllability at points $\mu_{0} \in P$, where the matrix $A\left(\mu_{0}\right)$ has blocks of the form $a^{3}$ or $a^{2} \beta^{2}$, by arbitrary small perturbation 
of one of the columns of $B(\mu)$. In the case of simple eigenvalues, this is as in the case $\operatorname{dim} P=1$. Now, it is obvious that analogously to the case $\operatorname{dim} P=1$, it is possible to prove the following theorem.

THEOREM 2. Let $r \geqslant 1, m>1$ and let $\operatorname{dim} P=2$. Then there is a subset $F_{3}^{r}(n, m) \subset F_{P}^{r}(n, m)$, residual in $F_{P}^{r}(n, m)$, such that if $(A, B) \in F_{3}^{r}(n, m)$, then the set $R(A, B)$ is a 1-dimensional submanifold of $P$.

Remark. If $m=1$, then the set $R(A, B)$ consists generically of 1-dimensional submanifolds and of isolated points.

Conjecture. If $\operatorname{dim} P=k$ and $m>k$, then there is a subset $H_{P}^{r}(n, m)$ $\subset F_{P}^{r}(n, m)$, residual in $F_{P}^{r}(n, m)$, such that for every $(A, B) \in H_{P}^{r}(n, m)$, we have $R(A, B)=\emptyset$.

\section{References}

[1] R. Abraham and J. Robbin, Transversal mappings and flows, Benjamin, New York 1967.

[2] В. И. Арнольд, Лекиии $\rho$ бифуркациях и версальных семействах, УМН 27: 5 (1972), p. 119-184.

[3] - O матрицах зависяцих от параметров, УМН 26: 2 (1971), p. 101-114.

[4] P. Brunovský, On one parameter families of diffeomorphisms, Comm. Math. Univ. Carolinae 12 (1971), p. 765-784.

[5] E. B. Lee and L. Markus, Foundation of optimal control theory, Russian trans-' lation, Nauka, Moscow 1972.

[6] R. Thom and H. Levine, Singularities of differentiable mappings, Russian translation, Mir, Moscow 1968. 